\title{
Inhibition of microRNA-155 modulates endotoxin tolerance by upregulating suppressor of cytokine signaling 1 in microglia
}

\author{
XIAOLEI SUN* , JIE SUN* , XIAOYI SHAO, JINRONG FENG, JUNMING YAN and YONGWEI QIN \\ Department of Pathogen Biology and Immunology, Medical College, Nantong University, Nantong, Jiangsu 226001, P.R. China
}

Received September 30, 2017; Accepted January 18, 2018

DOI: $10.3892 /$ etm.2018.6032

\begin{abstract}
Endotoxin tolerance is an immunohomeostatic reaction to reiterant lipopolysaccharide (LPS) exposure that maintains a state of altered responsiveness in immune cells, resulting in the inhibition of the pro-inflammatory response and the resolution of inflammation. Microglia constitutes the first line of defense against endogenous and external challenges in the brain. MicroRNAs (miRs) serve a critical function in the regulation of inflammation. The aim of the present study was to investigate whether miR-155 regulates endotoxin tolerance. miR-155 and suppressor of cytokine signaling-1 (SOCS1) mRNA expression was measured using RT-qPCR. The expression of SOCS1 was measured by western blotting and immunofluorescence. TNF- $\alpha$ levels were detected by an enzyme-linked immunosorbent assay. The results indicated that miR-155 expression was significantly downregulated in the microglia and cortex tissue following the induction of endotoxin tolerance. This was consistent with an increase in the expression of SOCS1, a predicted target of miR-155 and key inhibitor of the inflammatory reaction. Transfection with miR-155 inhibitor significantly enhanced SOCS1 expression in the microglia following the induction of endotoxin tolerance. SOCS1 knockdown using short hairpin RNA partly inhibited the anti-inflammatory process and promoted the inflammatory response during endotoxin tolerance. The results of the current study indicate that miR-155 inhibition contributes to the development of endotoxin tolerance. Understanding how miRs regulate inflammatory mechanisms may facilitate the development of novel therapeutic strategies to treat CNS disorders.
\end{abstract}

Correspondence to: Dr Yongwei Qin, Department of Pathogen Biology and Immunology, Medical College, Nantong University, 19 Qixiu Road, Nantong, Jiangsu 226001, P.R. China

E-mail: yw_qin@foxmail.com

*Contributed equally

Key words: endotoxin tolerance, suppressor of cytokine signaling 1, microRNA-155, BV2, microglia

\section{Introduction}

Sepsis-induced central nervous system (CNS) dysfunction is a potentially irreversible acute brain dysfunction; as many as $71 \%$ of such patients go onto develop septic encephalopathy (SE) (1). SE is caused by systemic inflammation induced by the immune response to lipopolysaccharide stimulation (LPS). The resident CNS macrophages, microglia, are the primary target cells of LPS and it has been demonstrated that sepsis induced by peripheral injection of LPS activates microglial cells $(2,3)$. The pathogenesis of SE may be influenced by various elements, including the local and systemic secretion of pro-inflammatory mediators, activation of microglia, alterations in homeostasis, neurotransmitter imbalances and the effects of sepsis-induced peripheral multiple organ failure (1). It has also been demonstrated that the systemic injection of LPS, either as a single or repeated injection, impacts the cognitive competence of rats (4).

LPS-induced pro-inflammatory molecules are vital for regulating the growth and dissemination of pathogens; however, overproduction of LPS may induce sepsis syndrome, also known as endotoxin shock (5). Prior exposure to low doses of LPS cause the cell to become resilient to subsequent LPS challenge; cells develop tolerance to endotoxins, which is known as endotoxin tolerance $(5,6)$. Endotoxin tolerance has been observed in vitro and in vivo, in animal models and in humans (5). It is also associated with changes in particular regulatory events, including deficiencies in the myeloid differentiation primary response 88 (MyD88) (7), reduced IL-1 receptor-associated kinase (IRAK)4-MyD88 association (8), suppressed IRAK1 activation (9), as well as the upregulation of negative regulators, including IRAK-M (10), suppressor of cytokine signaling 1 (SOCS1) and SH2 domain containing inositol-5-phosphatase (SHIP-1) (11). Furthermore, studies have indicated that nuclear factor (NF)-kB subunits (12), as well as peroxisome proliferator-activator receptor $\gamma(13)$ are involved in the development of endotoxin tolerance. It has also been demonstrated that SOCS1 affects a series of signaling pathways, including NF- $\mathrm{B}$ (14), c-Jun N terminal kinase and p38 (15).

In CNS cells, including microglia, oligodendrocytes, astrocytes and neurons, SOCS1 expression is induced by LPS, interferon (IFN) $-\beta$ or $-\gamma$, and interleukin (IL)- 4 or -6 . The effects of SOCS1 on neurological results vary greatly depending on the disease context and the neuroinflammatory 
microenvironment (16). SOCS1 ${ }^{-/-}$mice develop multiple organ failures (17). Additionally, macrophages from $\mathrm{SOCS1}^{-/}$mice are hypersensitive to toll-like receptor (TLR) ligands, as indicated by increases in the expression of pro-inflammatory chemokines and cytokines (18). It has been hypothesized that SOCS1 may be used as a novel therapeutic strategy to treat patients with multiple sclerosis (19) and different types of cancer (20). SOCS1 serves a vital role in modulating TLR-mediated responses and may therefore be used as a therapeutic regulator in CNS diseases.

However, the nature of the endotoxin tolerance that modulates SE remains unclear. Indeed, to the best of our knowledge, no experiments have addressed the effect of SOCS1 on microglia during the development of endotoxin tolerance.

microRNAs (miRs) are small noncoding RNAs that regulate multiple human biological processes via the posttranslational control of mRNA expression (21). A number of miRs modulate endotoxin tolerance $(22,23)$. It has been demonstrated that miR-155 serves crucial roles in the immune response, glioma, B-cell malignancies and hematopoiesis. The contribution of miR-155 to the development of endotoxin tolerance has also been demonstrated in humans and an endotoxin shock mouse model $(24,25)$. miR-155 targets and represses several downstream TLR4 mediators, including tumor necrosis factor (TNF)- $\alpha$, transcription factor PU.1, SHIP1, SOCS1, TNF receptor-associated factor-6, IRAK1 and interferon regulatory factor 5, highlighting miR-155 in the development of endotoxin tolerance (24).

The present study measured the expression of SOCS1 and miR-155 in microglia and the murine cortex under states of inflammation and endotoxin tolerance, and investigated the function of miR-155 in modulating endotoxin tolerance in microglia. The results of the current study demonstrated that endotoxin tolerance induced the downregulation of miR-155 and upregulated the expression of SOCS1, thus decreasing the production of pro-inflammatory TNF- $\alpha$. This suggests that miR-155 may be used to regulate inflammation and endotoxin tolerance in microglia.

\section{Materials and methods}

Cell culture. BV2 cells (American Type Culture Collection, Manassas, VA, USA) were cultured in Dulbecco's modified Eagle's medium (DMEM)-F12 (Thermo Fisher Scientific, Inc., Waltham, MA, USA) supplemented with $10 \%$ fetal calf serum (ScienCell Research Laboratories, Inc., Carlsbad, CA, USA), in a $5 \% \mathrm{CO}_{2}$ humidified atmosphere at $37^{\circ} \mathrm{C}$. Primary mouse (C57BL/6; Experimental Animal Center of Nantong University, Nantong, China) microglial cultures were cultured following a previously described protocol (26).

Cell transfections. BV2 cell transfections of $200 \mathrm{nM}$ miR-155 mimic (UUAAUGCUAAUUGUGAUAGGGGU, double strand), miR-155 inhibitor (AAUUACGAUUAACACUAU CCCCA, single strand), mimic negative control (NC) (UUC UCCGAACGUGUCACGU, double strand) and inhibitor negative control (AAGAGGCUUGCACAGUGCA, single strand; all Guangzhou RiboBio Co., Ltd., Guangzhou, China) were performed using HiPerFect Transfection Reagent (Qiagen, Inc., Valencia, CA, USA) following the manufacturer's protocol. The following day, the medium was changed and cells were subjected to single LPS (defined as LPS) or repeated LPS (defined as LPS/LPS) challenge.

Cell tolerance model. To induce endotoxin tolerance, BV2 cells were treated with $10 \mathrm{ng} / \mathrm{ml}$ LPS (Sigma-Aldrich; Merck KGaA, Darmstadt, Germany) for $18 \mathrm{~h}$, followed by washout of LPS with PBS. Cells were rested for a further $2 \mathrm{~h}$ prior to restimulation with $100 \mathrm{ng} / \mathrm{ml}$ LPS for $4,6,8$ or $10 \mathrm{~h}$. To induce inflammation, BV2 cells were treated with a single stimulation of $100 \mathrm{ng} / \mathrm{ml}$ LPS for 4 or $8 \mathrm{~h}$ (prior to mRNA detection), or for 6 or $10 \mathrm{~h}$ (for protein detection). RNA, protein and cell-free supernatants were collected at indicated times, following the final stimulation, respectively.

Murine tolerance model. A total of $28 \mathrm{C} 57 \mathrm{BL} / 6$ mice (weighing 22-25 g; 4 mice/group; 14 males and 14 females) were obtained from Experimental Animal Center of Nantong University, given access to regular chow and sterile water at regaular intervals, and housed at $21-23^{\circ} \mathrm{C}$ with relative humidity of $50 \pm 5 \%$ on a $12 \mathrm{~h}$ light cycle. To induce endotoxin tolerance, the C57BL/6 mice were injected intraperitoneally (i.p.) with an initial dose of $5 \mathrm{mg} / \mathrm{kg}$ LPS. After 3 days, mice were re-challenged with $10 \mathrm{mg} / \mathrm{kg}$ LPS (i.p.) on day 1,3 and 7. To induce inflammation, mice received an i.p. injection of $200 \mu \mathrm{l}$ PBS 3 days prior to $10 \mathrm{mg} / \mathrm{kg}$ LPS administration for 1, 3 and 7 days. Mice were sacrificed on the day of their respective final injections. Tissues were collected and stored at $-80^{\circ} \mathrm{C}$ until use. All animal experiments were performed in accordance with the Guide for the Care and Use of Laboratory Animals (27) and were approved by the Ethics Committee of the Nantong University (Nantong, China).

SOCS1 knockdown. Non-sense short interfering (sh)RNA (scramble), SOCS1 shRNA1 and SOCS1 shRNA2 (Table I) were designed by using the Sigma online database (https://www. sigmaaldrich.com/china-mainland/zh/life-science/functionalgenomics-and-rnai/shrna/individual-genes.html) of validated shRNAs. shRNAs $(1 \mu \mathrm{g})$ were subcloned between the BamHI and NotI site of the pGreenPuro shRNA Expression Lentivector (System Biosciences, Palo Alto, CA, USA). BV2 cells were transfected into the plasmid using the HiPerFect Transfection Reagent following the manufacturer's protocol and $12 \mathrm{~h}$ after transfection, cells were subjected to LPS or LPS/LPS treatment.

RNA isolation and reverse transfection-quantitative polymerase chain reaction ( $R T-q P C R)$. Total RNAs were extracted from cell cultures and mice tissues using TRIzol (Thermo Fisher Scientific, Inc.). cDNAs were synthesized using M-MuLV Reverse Transcriptase in the QuantiTect ${ }^{\circledR}$ Reverse Transcription kit (Qiagen, Inc.) for $15 \mathrm{~min}$ at $42^{\circ} \mathrm{C}$. qPCR was performed on a CFX96 Touch $^{\mathrm{TM}}$ System Real-Time PCR Detection system (Bio-Rad Laboratories, Inc., Hercules, CA, USA), using SYBR-Green (Takara, Bio, Inc., Otsu, Japan). The thermocycling conditions were as follows: 40 Cycles of denaturation at $94^{\circ} \mathrm{C}$ for $15 \mathrm{sec}$, annealing at $60^{\circ} \mathrm{C}$ for $15 \mathrm{sec}$ and extension at $72^{\circ} \mathrm{C}$ for $10 \mathrm{sec}$. The relative amount of transcripts was calculated using the $2^{-\Delta \Delta \mathrm{Cq}}$ method (28). GAPDH and RNU6-1 were used as housekeeping genes. SOCS1 was 
Table I. shRNA sequences.

\begin{tabular}{ll}
\hline Gene & \\
\hline SOCS1 shRNA1 & F: gatccGCGCGACAGTCGCCAACGGAATTCAAGAGATTCCGTTGGCGACTGTCGCGCTTTTTg \\
& R: aattCAAAAACACCCTGCGGAACTTGTTCAATCTCTTGAATTGAACAAGTTCCGCAGGGTGg \\
SOCS1 shRNA2 & F: gatccGCGAGACCTTCGACTGCCTTTTTCAAGAGAAAAGGCAGTCGAAGGTCTCGCTTTTTg \\
& R: aattCAAAAAGCGAGACCTTCGACTGCCTTTTCTCTTGAAAAAGGCAGTCGAAGGTCTCGCg \\
Scramble shRNA & F: gatccTTCTCCGAACGTGTCACGTTTCAAGAGAACGTGACACGTTCGGAGAATTTTTg \\
& R: aattCAAAAATTCTCCGAACGTGTCACGTTCTCTTGAAACGTGACACGTTCGGAGAAg
\end{tabular}

Lowercase letters represent the restriction enzyme cutting site. sh, short hairpin; SOCS1, suppressor of cytokine signaling 1; F, forward; R, reserve.

normalized to GAPDH and miR-155 was normalized to RNU6-1. All primers used for the RT-qPCR analysis are listed in Table II.

Western blotting. Cells were lysed in a lysis buffer $(20 \mathrm{mM}$ Hepes, 120 mM NaCl, 2 mM EDTA, 1\% Nonidet P-40, $1 \%$ sodium deoxycholate, $0.1 \%$ SDS, protease inhibitor). Cellular debris was removed by centrifugation at $15,000 \mathrm{x} \mathrm{g}$ at $4^{\circ} \mathrm{C}$ for $30 \mathrm{~min}$. The protein concentrations in each sample were determined using NanoDrop 1000 (Thermo Fisher Scientific, Inc.). A total of $80 \mu \mathrm{g}$ /lane were resolved on $10 \% \mathrm{SDS}-\mathrm{PAGE}$ gels, transferred onto the polyvinylidene difluoride membrane and blocked in 5\% fat-free milk at room temperature for $2 \mathrm{~h}$. Membranes were then immunoblotted with anti-SOCS1 (1:500; cat. no. ab9870; Abcam, Cambridge, UK) or anti- $\beta$-actin (1:4,000; cat. no. 4970; Cell Signaling Technology, Inc., Danvars, MA, USA) antibodies at $4^{\circ} \mathrm{C}$ overnight. Subsequently, membranes were washed three times with PBS for 5 min each wash and incubated with anti-goat (1:2,000; cat. no. sc-2020; Santa Cruz Biotechnology, Inc., Dallas, TX, USA) or anti-rabbit (1:5,000; cat. no. 7074; both Cell Signaling Technology, Inc.) horseradish peroxidase-conjugated immunoglobulin $\mathrm{G}$ at $4^{\circ} \mathrm{C}$ for $2 \mathrm{~h}$. Blots were developed using an enhanced chemiluminescence kit (EMD Millipore, Billerica, MA, USA). The density of bands was measured using ImageJ $1.42 q$ software (National Institutes of Health, Bethesda, MD, USA).

Immunocytochemistry. BV2 cells were fixed on coverslips with $4 \%$ paraformaldehyde for $0.5 \mathrm{~h}$ at room temperature, followed by membrane permeabilization using $0.1 \%$ Triton $\mathrm{X}-100$. Cells were then blocked with $5 \%$ bovine serum albumin (Sigma-Aldrich; Merck KGaA) at room temperature for $2 \mathrm{~h}$, and incubated with primary antibodies against SOCS1 (1:100; cat. no. ab9870; Abcam) and cluster of differentiation 11b (1:100; cat. no. ab128797; Abcam) at $4^{\circ} \mathrm{C}$ overnight. Cells were then incubated with anti-goat immunoglobulin (Ig)G conjugated with tetramethylrhodamine (1:2,000; cat. no. ab6882; Abcam) or anti-rabbit IgG conjugated with fluorescein isothiocyanate secondary antibodies (1:2,000; cat. no. ab6798; Abcam) at $4^{\circ} \mathrm{C}$ for $2 \mathrm{~h}$. Coverslips were mounted on a slide with glycerol and cells were viewed using a confocal microscope.

Enzyme-linked immunosorbent assay (ELISA). BV2 were incubated for $24 \mathrm{~h}$ at $37^{\circ} \mathrm{C}$ in $5 \% \mathrm{CO}_{2}$, culture medium was
Table II. Primer sequences.

\begin{tabular}{|c|c|}
\hline Gene & Sequences $\left(5^{\prime}-3^{\prime}\right)$ \\
\hline \multirow[t]{2}{*}{ SOCS1 } & Forward: GAGACCTTCGACTGCCTTTTC \\
\hline & Reverse:TAGTCACGGAGTACCGGGTTAAG \\
\hline \multirow[t]{2}{*}{ GAPDH } & Forward: TGACCTCAACTACATGGTCTACA \\
\hline & Reverse: CTTCCCATTCTCGGCCTTG \\
\hline \multirow[t]{4}{*}{$\operatorname{miR}-155$} & RT: GTCGTATCCAGTGCAGGGTCCGAGG \\
\hline & TATTCGCACTGGATACGACACCCCT \\
\hline & Forward: CGCCTGTTAATGCTAATTGTGA \\
\hline & Reverse: AGTGCAGGGTCCGAGGTAT \\
\hline \multirow[t]{3}{*}{ RNU6-1 } & RT: AACGCTTCACGAATTTGCGT \\
\hline & Forward: CTCGCTTCGGCAGCACA \\
\hline & Reverse: AACGCTTCACGAATTTGCGT \\
\hline
\end{tabular}

RNU6-1, RNA U6 small nuclear 1; SOCS1, suppressor of cytokine signaling 1 ; miR, microRNA.

collected and centrifuged at $700 \mathrm{x}$ g at $4^{\circ} \mathrm{C}$ for $5 \mathrm{~min}$. Cell-free supernatants were transferred to 96 well plate and TNF- $\alpha$ levels were measured using an ELISA kit (cat. no. 560478; BD Biosciences, San Jose, CA, USA) following the manufacturer's protocol.

Statistical analysis. Results were evaluated for statistical significance using GraphPad Prism 5 (GraphPad software, Inc., La Jolla, CA, USA). All data are presented as the mean \pm standard error of the mean. Two-way analysis of variance was performed, followed by Turkey's HSD post hoc tests; these tests were used to determine whether differences among groups were significant and $\mathrm{P}<0.05$ was determined to indicate a significant difference.

\section{Results}

The effects of inflammation and endotoxin tolerance on miR-155 induction in murine microglia and the mouse cortex. To investigate and compare miR-155 expression in microglia during inflammation and endotoxin tolerance, BV2 cells and primary microglia were treated with LPS to induce inflammation or repeated LPS to induce endotoxin tolerance. miR-155 
A

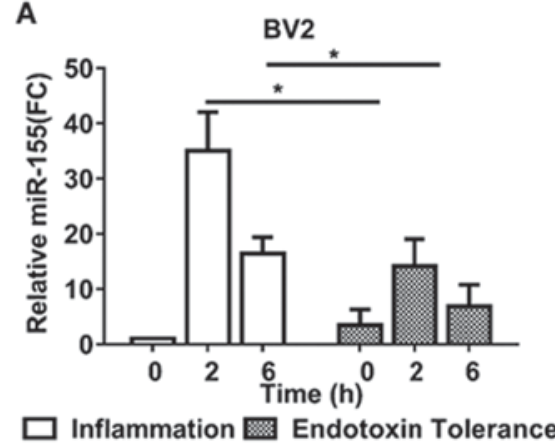

B

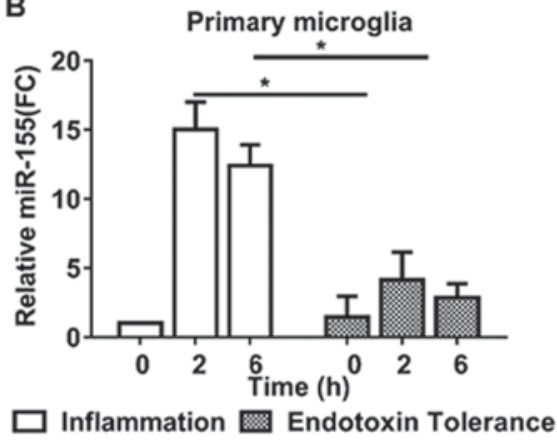

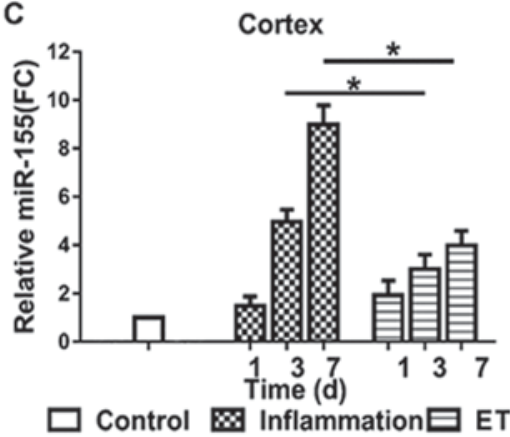

Figure 1. Expression of miR-155 in the BV2 cells, primary microglia and the mouse cortex. miR-155 expression was measured in (A) BV2 and (B) primary microglia following treatment with $100 \mathrm{ng} / \mathrm{ml}$ LPS alone or $10 \mathrm{ng} / \mathrm{ml} \mathrm{LPS}$ and then $100 \mathrm{ng} / \mathrm{ml} \mathrm{LPS} \mathrm{for} 2$ or $6 \mathrm{~h}$. (C) miR-155 expression in the cortex was also measured. Tissue from the cortices of inflammatory and endotoxin tolerant mice were extracted at the indicated times (1, 3 and 7 days). miR-155 expression was measured using reverse transcription-quantitative polymerase chain reaction. Experiments were performed at least three biologically independent replicates and data are presented as the mean \pm standard error of the mean. ${ }^{*} \mathrm{P}<0.05$. miR, microRNA; LPS, lipopolysaccharide.

expression was measured using RT-qPCR. miR-155 expression reached a peak at $2 \mathrm{~h}$ and subsequently declined in inflammatory BV2 (Fig. 1A) and primary microglia (Fig. 1B). Following treatment with repeated LPS, cells became endotoxin tolerant; miR-155 expression did not increase significantly following further stimulation in tolerant BV2 (Fig. 1A) and primary microglia (Fig. 1B).

To evaluate the expression of miR-155 in inflammatory and endotoxin tolerant mouse microglia in vivo, miR-155 levels in mouse cortex tissue were evaluated. Treatment of mice with single LPS to induce inflammation produced similar results to those of BV2 and primary microglia; mice exhibited a 4- and 8-fold upregulation in miR-155 expression 3 and 7 days following LPS administration, respectively. However, following treatment of mice with repeated LPS to induce endotoxin tolerance, miR-155 expression was only moderately upregulated in the 7 days following subsequent LPS administration (Fig. 1C). These results indicate that miR-155 serves a potential modulatory role during endotoxin tolerance.

The effects of inflammation and endotoxin tolerance on SOCS1 expression in murine microglia and cortex. The aim of the present study was to clarify the role of miR-155 in modulating endotoxin tolerance and whether it did this by affecting SOCS1 expression. Therefore, the expression of SOCS1 in murine microglial cells and the cortices of mice following the induction of inflammation and endotoxin tolerance, respectively, were evaluated (Fig. 2). Following the induction of endotoxin tolerance, the expression of SOCS1 mRNA (Fig. 2A) and protein (Fig. 2C and E) were significantly increased in the mouse cortex, compared with mice that only exhibited inflammation. Furthermore, BV2 cells treated with repeated LPS exhibited increases in the expression of SOCS1 mRNA (Fig. 2B) and protein (Fig. 2D and F), compared with cells treated with LPS alone. These results indicate that SOCS1 is involved in modulating endotoxin tolerance in microglia.

Endotoxin tolerance mediates SOCS1 expression via miR-155 in murine microglia. TargetScan indicated that miR-155 is able to bind to the 3'-untranslated region of SOCS1 mRNA and previous studies have demonstrated that miR-155 affects SOCS1 expression in LPS-treated mouse N9 microglia and other immune cells (29-31). To examine whether miR-155 regulates the expression of SOCS1 during inflammation and endotoxin tolerance, the expression of SOCS1 in LPS-treated BV2 microglia was measured. Compared with the NC, the overexpression of miR-155 following transfection of miR-155 mimic significantly downregulated SOCS1 expression, whereas inhibition of miR-155 following transfection with miR-155 inhibitor significantly upregulated SOCS1 expression (Fig. 3A and B). The same results were identified following immunocytochemistry to determine the cytoplasmic expression of SOCS1 (Fig. 3C). These results are partly in accordance with the results of a study by Cardoso et al (31), which identified that SOCS1 is a target of miR-155 in N9 microglia. These results indicate that SOCS1 is a direct target of miR-155 in BV2 cells following treatment with LPS.

To further investigate the role of miR-155 in regulating the induction of endotoxin tolerance by SOCS1, miR-155 NC and miR-155 inhibitor were transfected in BV2 microglia prior to the induction of inflammation and endotoxin tolerance. The results demonstrated that the expression of SOCS1 was significantly upregulated in BV2 microglia that were endotoxin tolerant compared with those that had only undergone inflammation. This was the case in BV2 microglia transfected with miR-155 inhibitor or the NC (Fig. 3D and E). Furthermore, in BV2 microglia transfected with the miR-155 inhibitor, the expression of SOCS1 was significantly increased following the induction of endotoxin tolerance or inflammation, compared with those that had undergone transfection with the NC. Taken together, these results suggest that miR-155 inhibition maintains the state of endotoxin tolerance in BV2 microglia, at least partly, via SOCS1.

The role of SOCS1 in anti-inflammatory phenotype during endotoxin tolerance. To further investigate the role of miR-155 in regulating the SOCS1-induced microglia anti-inflammatory phenotype, loss-of-function experiments were performed. Cells transfected with an shRNA against SOCS1 was markedly (Fig. 4A) and significantly (Fig. 4B) low compared with cells transfected with a scrambles shRNA. 
A

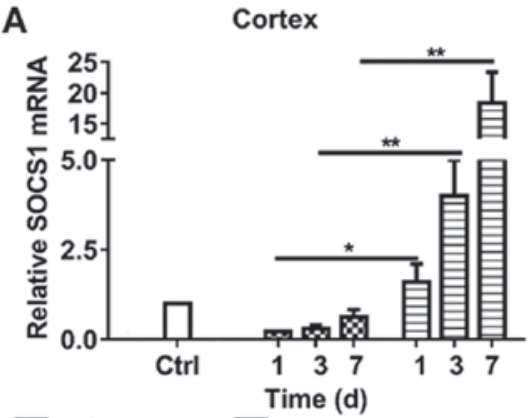

国 Inflammation $\mathrm{Q}$ Endotoxin Tolerance

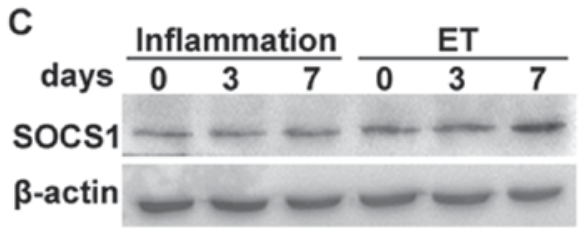

E

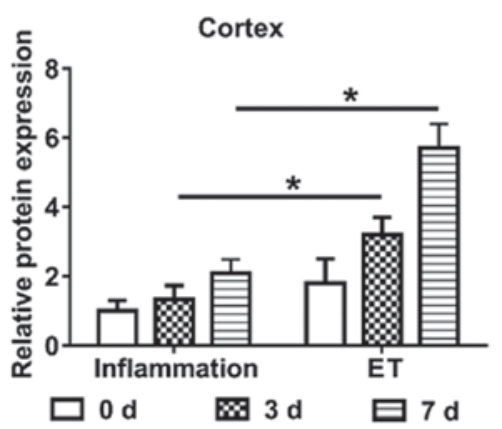

B

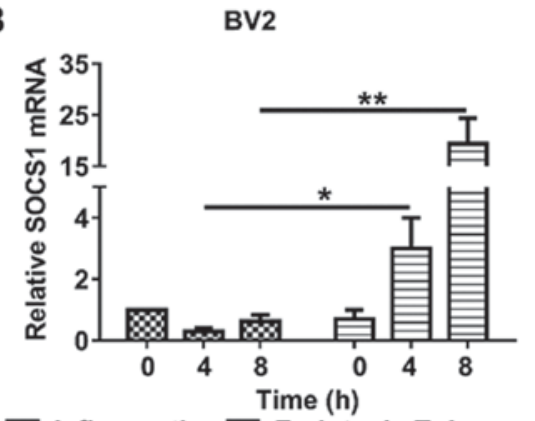

\$ Inflammation $\mathrm{O}$ Endotoxin Tolerance

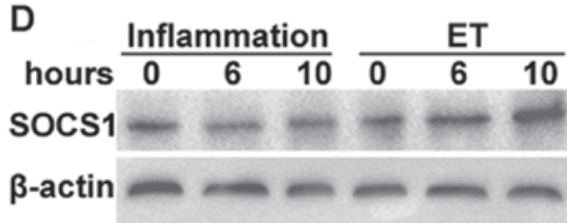

$F$

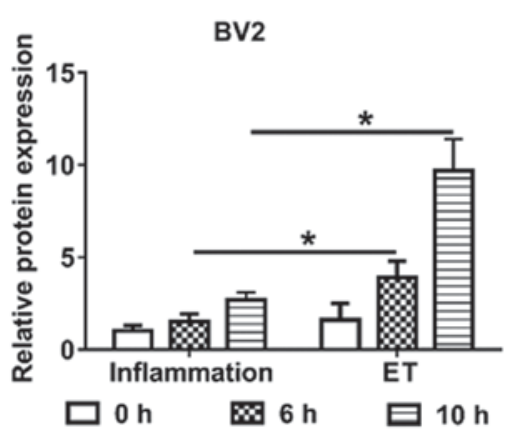

Figure 2. (A-F) Expression of SOCS1 in the mouse cortex and BV2 cells. To induce inflammation and ET, BV2 cells were treated with a single injection of $100 \mathrm{ng} / \mathrm{ml}$ LPS, or 10 or $100 \mathrm{ng} / \mathrm{ml}$ LPS, respectively. SOCS1 (A) mRNA and (C) protein expression were detected in the cortex. SOCS1 (B) mRNA and (D) protein expression were detected in BV2 cells. (E and F) Densitometric evaluation of SOCS1 band intensities using Image J. Each experiment was repeated at least three times. The results are presented as the mean \pm standard error of the mean. ${ }^{*} \mathrm{P}<0.05$ and ${ }^{* *} \mathrm{P}<0.01$. ET, endotoxin tolerance; SOCS1, suppressor of cytokine signaling 1 ; LPS, lipopolysaccharide.

It was demonstrated that LPS significantly increased TNF- $\alpha$ expression in cells transfected with SOCS1 shRNA1 and SOCS1 shRNA2 to knockdown SOCS1 expression compared with those transfected with scramble shRNA cells (Fig. 4C). However, in cells treated with LPS/LPS to induce endotoxin tolerance, the expression of TNF- $\alpha$ in cells transfected with scramble shRNA was significantly decreased compared with those transfected with SOCS1 shRNA. Notably, in cells transfected with shRNA SOCS1 to induce SOCS1 knockdown, LPS significantly increased TNF- $\alpha$ production compared with those treated with LPS/LPS (Fig. 4C). These data highlight the biological relevance of SOCS1 during the development of endotoxin tolerance; silencing SOCS1 restores the pro-inflammatory phenotype. Taken together, these results indicate that miR-155 inhibition promotes the anti-inflammatory phenotype and endotoxin tolerance in BV2 microglia and that this occurs by increasing the expression of SOCS1.

\section{Discussion}

Endotoxin tolerance has been extensively investigated in peripheral macrophages $(5,7,9,10)$; however, little is known about the microglia and miRs that are involved in the development of endotoxin tolerance. Various studies have identified that miR-155 serves an important in modulating the innate and adaptive immune response; furthermore, the deregulation of miR-155 has been implicated in a number of different diseases $(32,33)$. The present study demonstrated that miR-155 serves a role in regulating the production of SOCS1 and modulating endotoxin tolerance in BV2 microglia. This may improve understanding regarding the pathogenetic mechanisms by which miR regulates endotoxin tolerance.

A number of studies have demonstrated that SOCS1 serves important roles in the CNS (16). Several pathogens are able to induce the expression of SOCS proteins as a method of evading the IFN-mediated innate immune responses in CNS $(15,16)$. Pathogen-induced increases in SOCS expression in the CNS are beneficial for the host $(15,16)$. Furthermore, SOCS1 influences the CNS inflammatory response during infection (16). However, the function of SOCS proteins in CNS immune cells has not yet been investigated. The current study found that the expression of miR-155 was upregulated and that the expression of SOCS1 was downregulated in BV2 cells, primary microglia and mice cortices following exposure to LPS. These results are in accordance with the results of a previous study, which demonstrated that miR-155 was upregulated and SOCS1 expression was decreased in N9 cells (31).

miR-155 is processed from a non-coding transcript, the B-cell Integration Cluster, and serves important functions 

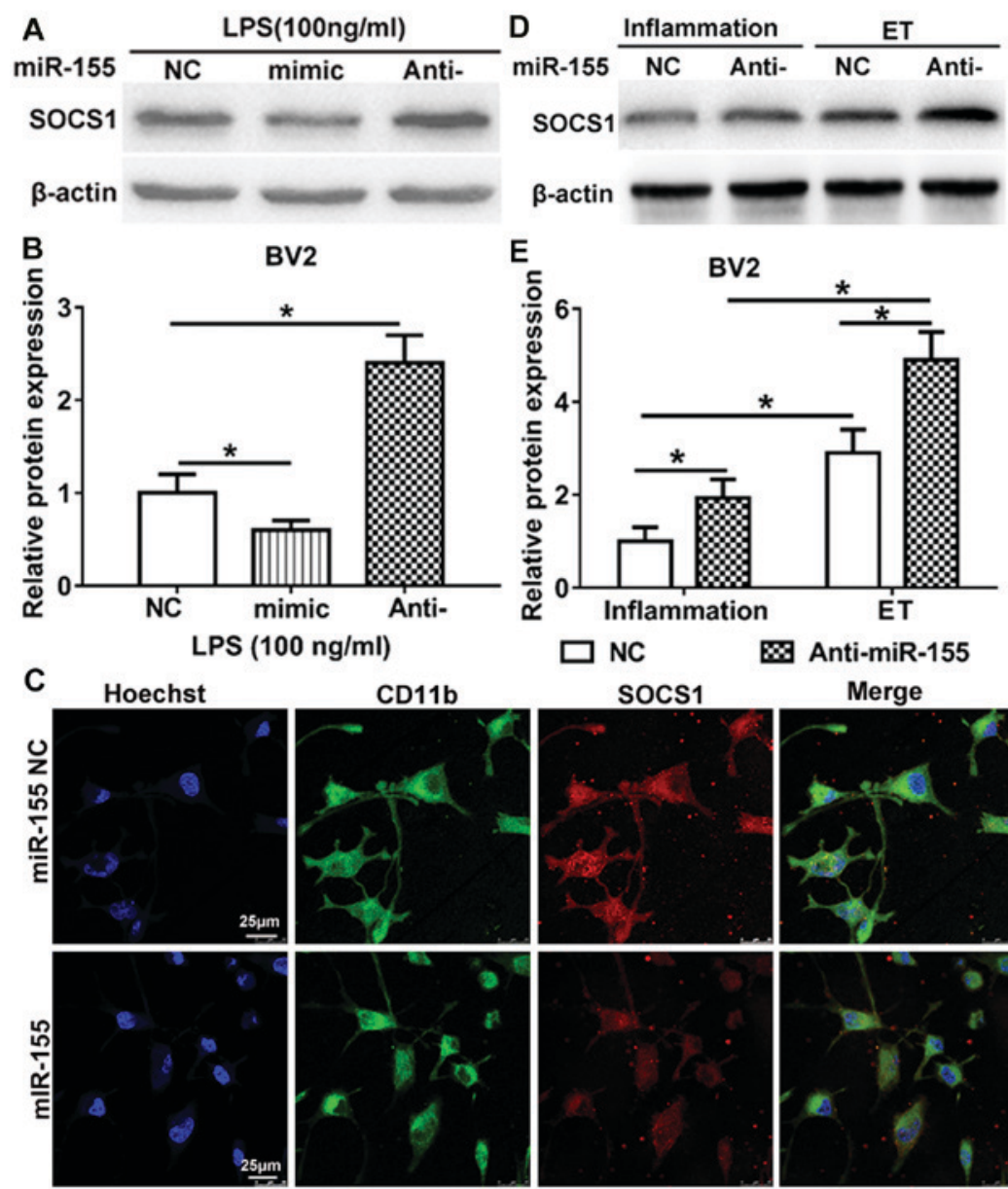

$\otimes$ Anti-miR-155
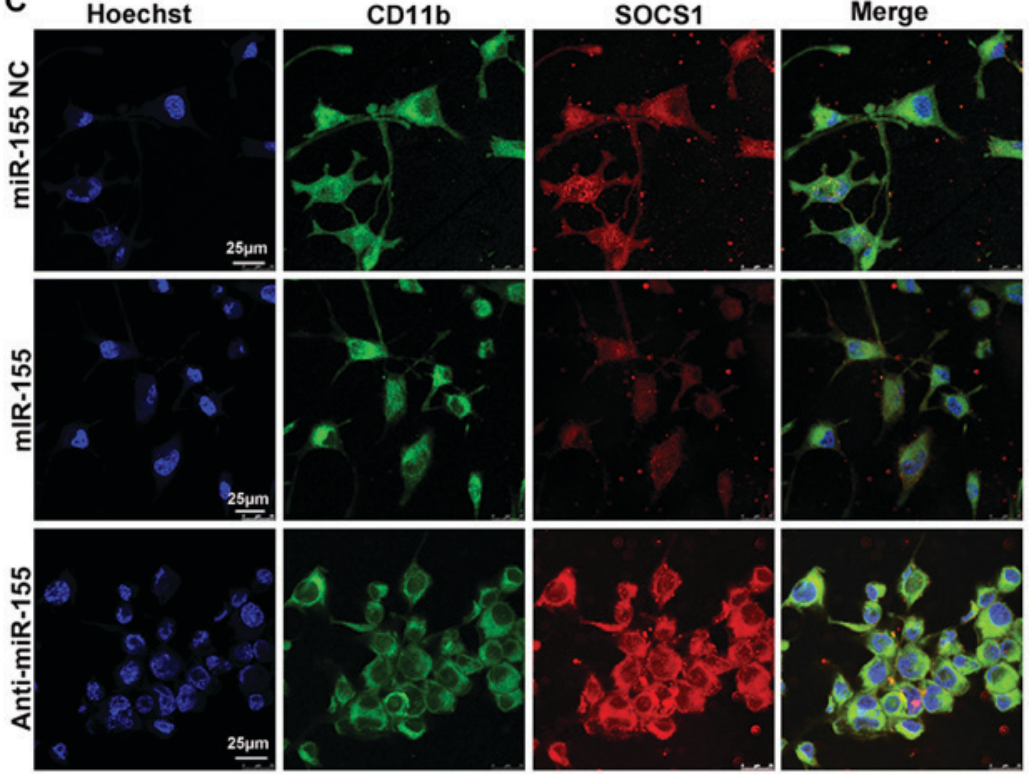

Figure 3. miR-155 inhibition promotes ET in BV2 microglia by induction of SOCS1. (A and B) Western blot analysis of SOCS1 expression in BV2 microglia. BV2 were transfected with miR-155 NC, miR-155 mimic or miR-155 inhibitor and subsequently treated with $100 \mathrm{ng} / \mathrm{ml}$ LPS to induce inflammation. (C) SOCS1 expression in the cytoplasm of BV2. Cells were transfected with miR-155 NC, miR-155 mimic, miR-155 inhibitor, and subsequently treated with $100 \mathrm{ng} / \mathrm{ml}$ LPS for $10 \mathrm{~h}$. SOCS1 (red) in the cytoplasm was detected by confocal microscopy. BV2 were stained with CD1lb (green). Nuclei of BV2 cells were stained with Hoechst (purple). (D) BV2 cells were transfected with miR-155 NC or miR-155 inhibitor and then treated with $100 \mathrm{ng} / \mathrm{ml} \mathrm{LPS}$ to induce inflammation or repeated 10-100 $\mathrm{ng} / \mathrm{ml}$ LPS to induce ET. SOCS1 expression in BV2 was detected by western blotting. (B and E) Densitometric evaluation of SOCS1 band intensities. Each experiment was repeated at least three times. The results are presented as the mean \pm standard error of the mean. $\mathrm{P}<0.05$. NC, negative control; LPS, lipopolysaccharide; ET, endotoxin tolerance; SOCS1, suppressor of cytokine signaling 1; CD, cluster of differentiation.
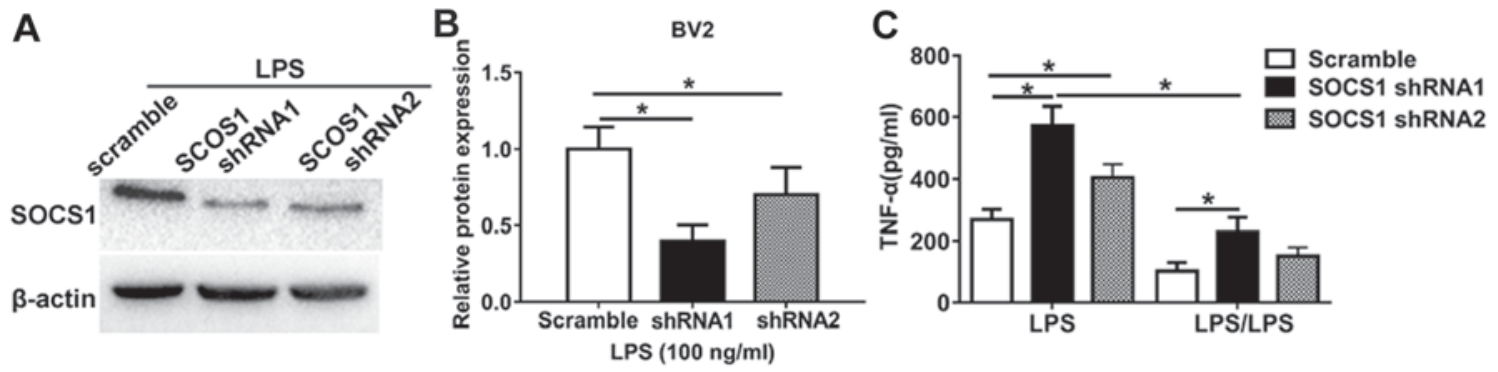

Figure 4. SOCS1 is involved in the induction of ET in BV2 microglia. (A) Western blotting to measure the shRNA-mediated knockdown of SOCS1 expression in BV2 cells. (B) Densitometric evaluation of SOCS1 band intensities. (C) BV2 cells transfected with either scramble shRNA, or SOCS1 shRNA1 and SOCS1 shRNA2 were subsequently treated with $100 \mathrm{ng} / \mathrm{ml}$ LPS to induce inflammation or repeated 10-100 ng/ml LPS to induce ET. Following $24 \mathrm{~h}$, supernatant TNF- $\alpha$ levels were measured using an enzyme-linked immunosorbent assay. All data are presented as the mean \pm standard error of the mean of three independent experiments. "P<0.05. sh, short hairpin; TNF, tumor necrosis factor; LPS, lipopolysaccharide; ET, endotoxin tolerance; SOCS1, suppressor of cytokine signaling 1. 
in cancer, the immune response and hematopoiesis (33). miR-155 expression is induced via TLRs in dendritic cells and macrophages and markedly affects the activities of these cells $(29,30)$. In microglia, the increase in miR-155 expression following activation may stimulate the production of pro-inflammatory mediators (34). It was demonstrated that decreases in miR-155 expression significantly reduced the expression of TNF- $\alpha$ and IL-6 (31). Wen et al (35) demonstrated that miR-155 promotes the inflammatory response by modulating TLR4/SOCS1 expression in ischemic cerebral tissues. Previous studies have also determined that miR-155 at least partly regulates endotoxin sensitivity and tolerance in macrophages $(24,36)$ and alveolar epithelial cells (37) by regulating SOCS1 expression. The results of the present study illustrate that miR-155 expression is downregulated in BV2 microglia, primary microglia and mice cortices following exposure to repeated LPS treatment. It was also demonstrated that SOCS1 mRNA and protein levels increase in BV2 and murine cells that exhibit endotoxin tolerance following repeated LPS treatment. Interestingly, the changes in SOCS1 expression following repeated LPS treatment were negatively associated with the change in miR-155 expression. Following transfection with miR-155 mimic and inhibitor, it was demonstrated that miR-155 negatively regulates SOCS1 expression in BV2 cells following exposure to LPS or repeated LPS.

SOCS1 is a vital negative regulator of cytokines, including TNF- $\alpha$ or IL- $1 \beta$ and therefore maintains the homeostasis of the immune system (15-18). Although microglial activation following pathological stimulation may be useful, it has been demonstrated that the failure to inhibit microglia-mediated immune responses at the appropriate time induces the overproduction of inflammatory mediators and results in the development of a chronic inflammatory state with severe consequences to ambient neurons (38). The results of the current study establish a direct link between miR-155 downregulation, upregulated SOCS1 expression and the inhibition of pro-inflammatory cytokines in BV2 cells following the development of endotoxin tolerance. This suggests that miR-155 inhibition may contribute to anti-inflammatory processes in the CNS by promoting the positive function of SOCS1 and decreasing levels of pro-inflammatory cytokines.

Taken together, the results of the current study demonstrate that miR-155 inhibitors are able to downregulate BV2 microglia mediated neuroinflammation by regulating SOCS1 expression and participate in sustaining endotoxin tolerance in CNS. These results may facilitate the development of valuable therapeutic strategies to treat CNS infections by decreasing inflammation and endotoxin tolerance using miRs.

\section{Acknowledgements}

Not applicable.

\section{Funding}

The present study was supported by the National Natural Science Foundation of China (grant no. 81201252). This project was also funded by the Scientific Research Programme of Nantong (grant no. MS12016007).

\section{Availability of data and materials}

The analyzed data sets generated during the study are available from the corresponding author on reasonable request.

\section{Authors' contributions}

$\mathrm{XSu}, \mathrm{YQ}$ and JS contributed to the conception and design of experiments. XSh, JF and JY conducted the experiments. XSu and YQ analyzed the data and drafted the manuscript. The final version of the manuscript has been read and approved by all authors, and each author believes that the manuscript represents honest work.

\section{Ethical approval}

All experiments were performed in accordance with the Guide for the Care and Use of Laboratory Animals and were approved by the Ethics Committee of the Nantong University (Nantong, China).

\section{Consent for publication}

Not applicable.

\section{Competing interests}

The authors declare that they have no competing interests.

\section{References}

1. Wilson JX and Young GB: Progress in clinical neurosciences: Sepsis-associated encephalopathy: Evolving concepts. Can J Neurol Sci 30: 98-105, 2003.

2. Buttini M, Limonta S and Boddeke HW: Peripheral administration of lipopolysaccharide induces activation of microglial cells in rat brain. Neurochem Int 29: 25-35, 1996.

3. Nimmerjahn A, Kirchhoff F and Helmchen F: Resting microglial cells are highly dynamic surveillants of brain parenchyma in vivo. Science 308: 1314-1318, 2005.

4. Sparkman NL, Kohman RA, Scott VJ and Boehm GW: Bacterial endotoxin-induced behavioral alterations in two variations of the Morris water maze. Physiol Behav 86: 244-251, 2005.

5. Biswas SK and Lopez-Collazo E: Endotoxin tolerance: New mechanisms, molecules and clinical significance. Trends Immunol 30: 475-487, 2009.

6. Foster SL, Hargreaves DC and Medzhitov R: Gene-specific control of inflammation by TLR-induced chromatin modifications. Nature 447: 972-978, 2007.

7. Medvedev AE, Lentschat A, Wahl LM, Golenbock DT and Vogel SN: Dysregulation of LPS-induced Toll-like receptor 4-MyD88 complex formation and IL-1 receptor-associated kinase 1 activation in endotoxin-tolerant cells. J Immunol 169: 5209-5216, 2002.

8. Li L, Cousart S, Hu J and McCall CE: Characterization of interleukin-1 receptor-associated kinase in normal and endotoxin-tolerant cells. J Biol Chem 275: 23340-23345, 2000.

9. Kobayashi K, Hernandez LD, Galán JE, Janeway CA Jr, Medzhitov R and Flavell RA: IRAK-M is a negative regulator of Toll-like receptor signaling. Cell 110: 191-202, 2002.

10. Zacharioudaki V, Androulidaki A, Arranz A, Vrentzos G, Margioris AN and Tsatsanis C: Adiponectin promotes endotoxin tolerance in macrophages by inducing IRAK-M expression. J Immunol 182: 6444-6451, 2009.

11. Piao W, Song C, Chen H, Diaz MA, Wahl LM, Fitzgerald KA, $\mathrm{Li} \mathrm{L}$ and Medvedev AE: Endotoxin tolerance dysregulates MyD88- and Toll/IL-1R domain-containing adapter inducing IFN-beta-dependent pathways and increases expression of negative regulators of TLR signaling. J Leukoc Biol 86: 863-875, 2009. 
12. Porta C, Rimoldi M, Raes G, Brys L, Ghezzi P, Di Liberto D, Dieli F, Ghisletti S, Natoli G, De Baetselier P, et al: Tolerance and M2 (alternative) macrophage polarization are related processes orchestrated by p50 nuclear factor kappaB. Proc Natl Acad Sci USA 106: 14978-14983, 2009.

13. Von Knethen AA and Brüne B: Delayed activation of PPARgamma by LPS and IFN-gamma attenuates the oxidative burst in macrophages. FASEB J 15: 535-544, 2001.

14. Ryo A, Suizu F, Yoshida Y,Perrem K, Liou YC, Wulf G, Rottapel R, Yamaoka S and Lu KP: Regulation of NF-kappaB signaling by Pin1-dependent prolyl isomerization and ubiquitin-mediated proteolysis of p65/RelA. Mol Cell 12: 1413-1426, 2003.

15. He Y, Zhang W, Zhang R, Zhang H and Min W: SOCS1 inhibits tumor necrosis factor-induced activation of ASK1-JNK inflammatory signaling by mediating ASK1 degradation. J Biol Chem 281: 5559-5566, 2006.

16. Baker BJ, Akhtar LN and Benveniste EN: SOCS1 and SOCS3 in the control of CNS immunity. Trends Immunol 30: 392-400, 2009.

17. Marine JC, Topham DJ, McKay C, Wang D, Parganas E, Stravopodis D, Yoshimura A and Ihle JN: SOCS1 deficiency causes a lymphocyte-dependent perinatal lethality. Cell 98: 609-616, 1999 .

18. Gingras S, Parganas E, de Pauw A, Ihle JN and Murray PJ: Re-examination of the role of suppressor of cytokine signaling 1 (SOCS1) in the regulation of toll-like receptor signaling. J Biol Chem 279: 54702-54707, 2004.

19. Mujtaba MG, Flowers LO, Patel CB, Patel RA, Haider MI and Johnson HM: Treatment of mice with the suppressor of cytokine signaling-1 mimetic peptide, tyrosine kinase inhibitor peptide, prevents development of the acute form of experimental allergic encephalomyelitis and induces stable remission in the chronic relapsing/remitting form. J Immunol 175: 5077-5086, 2005.

20. Huang FJ, Steeg PS, Price JE, Chiu WT, Chou PC, Xie K, Sawaya R and Huang S: Molecular basis for the critical role of suppressor of cytokine signaling-1 in melanoma brain metastasis. Cancer Res 68: 9634-9642, 2008.

21. Huntzinger E and Izaurralde E: Gene silencing by microRNAs: Contributions of translational repression and mRNA decay. Nat Rev Genet 12: 99-110, 2011.

22. Quinn EM, Wang J and Redmond HP: The emerging role of microRNA in regulation of endotoxin tolerance. J Leukoc Biol 91: 721-727, 2012.

23. Nahid MA, Satoh M and Chan EK: MicroRNA in TLR signaling and endotoxin tolerance. Cell Mol Immunol 8: 388-403, 2011.

24. Androulidaki A, Iliopoulos D, Arranz A, Doxaki C, Schworer S, Zacharioudaki V, Margioris AN, Tsichlis PN and Tsatsanis C: The kinase Akt1 controls macrophage response to lipopolysaccharide by regulating microRNAs. Immunity 31: 220-231, 2009.

25. Alexander M, Ramstead AG, Bauer KM, Lee SH, Runtsch MC, Wallace J, Huffaker TB, Larsen DK, Tolmachova T, Seabra MC, et al: Rab27-dependent exosome production inhibits chronic inflammation and enables acute responses to inflammatory stimuli. J Immunol 199: 3559-3570, 2017.
26. Saura J, Tusell JM and Serratosa J: High-yield isolation of murine microglia by mild trypsinization. Glia 44: 183-189, 2003.

27. National Research Council (US) Committee for the Update of the Guide for the Care and Use of Laboratory Animals. Guide for the Care and Use of Laboratory Animals. 8th edition. Washington (DC), National Academies Press (US), ISBN-13 978-0-309-15400-0, 2011.

28. Livak KJ and Schmittgen TD: Analysis of relative gene expression data using real-time quantitative PCR and the 2(-Delta Delta C(T)) method. Methods 25: 402-408, 2001

29. O'Connell RM, Chaudhuri AA, Rao DS and Baltimore D: Inositol phosphatase SHIP1 is a primary target of miR-155. Proc Natl Acad Sci USA 106: 7113-7118, 2009.

30. O'Connell RM, Taganov KD, Boldin MP, Cheng G and Baltimore D: MicroRNA-155 is induced during the macrophage inflammatory response. Proc Natl Acad Sci USA 104: 1604-1609, 2007.

31. Cardoso AL, Guedes JR, Pereira de Almeida L and Pedroso de Lima MC: miR-155 modulates microglia-mediated immune response by down-regulating SOCS-1 and promoting cytokine and nitric oxide production. Immunology 135: 73-88, 2012.

32. Staedel C and Darfeuille F: MicroRNAs and bacterial infection. Cell Microbiol 15: 1496-1507, 2013.

33. de Yébenes VG, Bartolomé-Izquierdo $\mathrm{N}$ and Ramiro AR: Regulation of B-cell development and function by microRNAs. Immunol Rev 253: 25-39, 2013.

34. Kumar A, Stoica BA, Loane DJ, Yang M, Abulwerdi G, Khan N, Kumar A, Thom SR and Faden AI: Microglial-derived microparticles mediate neuroinflammation after traumatic brain injury. J Neuroinflammation 14: 47, 2017.

35. Wen Y, Zhang X, Dong L, Zhao J, Zhang C and Zhu C: Acetylbritannilactone modulates MicroRNA-155-mediated inflammatory response in ischemic cerebral tissues. Mol Med 21: 197-209, 2015.

36. Doxaki C, Kampranis SC, Eliopoulos AG, Spilianakis C and Tsatsanis C: Coordinated regulation of miR-155 and miR-146a genes during induction of endotoxin tolerance in macrophages. J Immunol 195: 5750-5761, 2015.

37. Neagos J, Standiford TJ, Newstead MW, Zeng X, Huang SK and Ballinger MN: Epigenetic regulation of tolerance to toll-like receptor ligands in alveolar epithelial cells. Am J Respir Cell Mol Biol 53: 872-881, 2015.

38. Prinz $\mathrm{M}$ and Priller J: Microglia and brain macrophages in the molecular age: From origin to neuropsychiatric disease. Nat Rev Neurosci 15: 300-312, 2014.

(7) This work is licensed under a Creative Commons Attribution 4.0 International (CC BY 4.0) License. 